\title{
Caenodelphax teapae (Fowler) (Hemiptera: Delphacidae): nuevo vector natural del Mal de Río Cuarto virus (MRCV, Fijivirus) en Argentina
}

\author{
Velazquez, P.D.; A.M.M. de Remes Lenicov y G.A. Truol
}

\begin{abstract}
RESUMEN
El Mal de Río Cuarto virus (MRCV) es el agente causal del mal de Río Cuarto, una de las principales enfermedades del maíz en Argentina. Es transmitido en la naturaleza principalmente por Delphacodes kuscheli (Insecta: Hemiptera). La presencia de otros delfácidos en áreas afectadas por la enfermedad, permite suponer que éstos tendrían un rol en su epidemiología. En prospecciones realizadas entre los años 2003 y 2006 en las provincias de Tucumán y Salta (noroeste argentino), Caenodelphax teapae se destacó por ser una de las especies más abundantes en la vegetación espontánea. El MRCV fue transmitido experimentalmente a trigo a partir de ejemplares naturalmente infectados provenientes de campo en Tucumán. La reacción del virus en las pruebas serológicas realizadas a insectos y plantas infectadas, así como la sintomatología observada en éstas y la presencia de agregados de partículas virales en células floemáticas, confirmaron que $C$. teapae tuvo la capacidad de transmitir el MRCV a trigo desde poblaciones naturales capturadas en la provincia de Tucumán. Las evidencias demostraron por primera vez que las poblaciones naturales de $C$. teapae son portadoras del MRCV y que actúan como vectoras del virus con importantes implicancias en la epidemiología del mal de Río Cuarto.
\end{abstract}

Palabras clave: Mal de Río Cuarto virus, Fijivirus, Caenodelphax teapae, vector natural.

Velazquez, P.D.; A.M.M. de Remes Lenicov and G.A. Truol 2017. Caenodelphax teapae (Fowler) (Hemiptera: Delphacidae): a new natural vector of the Mal de Río Cuarto virus (MRCV, Fijivirus) in Argentina. Agriscientia 34: 39-45

\section{SUMMARY}

Mal de Río Cuarto virus (MRCV) is the causal agent of Mal de Río Cuarto, one of the major diseases of maize in Argentina. It is spread under natural conditions by Delphacodes kuscheli (Insecta: Hemiptera). The presence of other delphacids in areas affected by the disease suggests that they have a role in 
its epidemiology. In prospective surveys conducted between 2003 and 2006 in the provinces of Tucumán and Salta (northwestern Argentina), Caenodelphax teapae stood out as one of the most abundant species in wild vegetation. The MRCV was experimentally transmitted to wheat through naturally field-infected specimens from Tucumán. The reaction of the virus in serological tests on insects and infected plants as well as the observed symptoms, together with the presence of aggregates of viral particles in phloem cells, confirmed that $C$. teapae had the ability to transmit the MRCV to wheat from natural populations captured in the province of Tucumán. Evidence showed for the first time that natural populations of $C$. teapae carry the MRCV and act as vectors of the virus with important implications for the epidemiology of this disease.

Key words: Mal de Río Cuarto virus, Fijivirus, Caenodelphax teapae, natural vector.

P.D. Velazquez: Estación Experimental Agropecuaria Paraná, Instituto Nacional de Tecnología Agropecuaria (INTA), Ruta Provincial No $11 \mathrm{Km}$ 12 1/2, 3101 Oro Verde, Argentina. A.M.M. de Remes Lenicov: Departamento de Entomología, Facultad de Ciencias Naturales y Museo de La Plata, Universidad Nacional de La Plata, Paseo del Bosque s/n, 1900 La Plata, Argentina. G.A. Truol: Instituto de Patología Vegetal (IPAVE), Centro de Investigaciones Agropecuarias (CIAP), INTA, Camino 60 Cuadras Km 5 1⁄2, X5020ICA Córdoba, Argentina. Correspondencia a: velazquez.pablo@inta.gob.ar

\section{INTRODUCCIÓN}

El mal de Río Cuarto es la enfermedad viral más importante del maíz (Zea mays L.) en Argentina (Argüello Caro et al., 2013). Su agente causal es el Mal de Río Cuarto virus (MRCV), un virus perteneciente al género Fijivirus de la familia Reoviridae (Distéfano et al., 2002). Los síntomas típicos de la enfermedad incluyen enanismo de plantas, acortamiento de entrenudos, reducción del área foliar, atrofia de panojas y espigas, y presencia de rugosidades prominentes o enaciones sobre las nervaduras en el envés de las hojas (Nome et al., 1981; March et al., 1995; Lenardón et al., 1998).

La enfermedad estuvo inicialmente restringida a cultivos cercanos al área endémica (Río Cuarto, sur de la provincia de Córdoba), sin embargo, las prospecciones que se fueron realizando demostraron que estaba presente en otras áreas (Laguna et al., 2002). En la campaña agrícola 1996/1997 ocurrió la epidemia más importante del mal de Río Cuarto con pérdidas estimadas en 120 millones de dólares (Lenardón et al., 1998). Además de ocasionar una menor producción de grano, la virosis reduce la producción de materia verde para silaje y el volumen de rastrojo, los cuales constituyen una importante reserva forrajera invernal en los sistemas agrícola-ganaderos del sur de la provincia de Córdoba (Lenardón et al., 1999).

Los miembros del género Fijivirus son transmiti- dos a poáceas exclusivamente por insectos de la familia Delphacidae (Insecta: Hemiptera) de forma persistente propagativa (Francki et al., 1985). El principal vector del MRCV en la naturaleza es Delphacodes kuscheli Fennah (Remes Lenicov et al., 1985). Este virus, al igual que otros miembros del grupo, se halla confinado al tejido floemático de las plantas infectadas (Nome et al., 1981; Truol et al., 2001; Arneodo et al., 2002b) y luego que ha sido adquirido por D. kuscheli, el vector permanece infectivo (transmisor) y es capaz de transmitirlo hasta su muerte (Arneodo et al., 2002a). Esta capacidad de transmitir que adquiere el insecto se denomina infectividad. De este modo, el insecto cumple la función de reservorio del virus adquiriendo una gran importancia desde el punto de vista epidemiológico.

Otras especies de delfácidos son mencionadas como vectores naturales del MRCV, entre ellas Chionomus haywardi (Muir) (= Delphacodes haywardi Muir) (Velazquez et al., 2003) y Metade/phax propinqua (Fieber) (= Toya propinqua Fieber) (Mattio et al., 2005), esta última citada inicialmente también como experimental (Velazquez et al., 2001). Asimismo, se ha demostrado la transmisión experimental del virus en las especies Peregrinus maidis (Ashmead) (Virla et al., 2003), Pyrophagus tigrinus Remes Lenicov \& Varela (Velazquez et al., 2006; Remes Lenicov \& Varela, 2014b) y Tagosodes orizicolus (Muir) (Mattio et al., 2008). 
Además del maíz, el MRCV ha sido detectado en un amplio rango de poáceas, de las cuales las más importantes desde el punto de vista epidemiológico son el trigo (Triticum aestivum L.), la avena (Avena sativa L.), la cebada (Hordeum vulgare L.), el centeno (Secale cereale L.) y varias malezas y pasturas estivales (Conci et al., 1992; Ornaghi et al., 1993b; March et al., 1995; Rodríguez Pardina et al., 1998; Laguna et al., 2000). Aunque el virus no causa daños de importancia en los cereales de invierno, éstos cumplen un rol clave en el ciclo de la enfermedad al actuar como hospedantes de $D$. kuscheli y constituir una importante fuente de inóculo para la transmisión al maíz (Remes Lenicov et al., 1985, 1991; Virla \& Remes Lenicov, 1991; Ornaghi et al., 1993a, 1999; March et al., 1995; Brentassi \& Remes Lenicov, 1999).

Prospecciones realizadas entre los años 2003 y 2006 en Tucumán y Salta (noroeste de Argentina - NOA), provincias en donde ha sido citada la presencia del MRCV (Laguna \& Giménez Pecci, 2004), determinaron que Caenodelphax teapae Fowler fue la especie más frecuentemente representada luego de M. propinqua (Velazquez, 2010). C. teapae es una especie ampliamente distribuida y muy común en el Neotrópico, desde Estados Unidos (Florida y Texas), Caribe (Antigua, Cuba, Dominica, República Dominicana, Granada, Guadalupe, Haití, Islas Caimán, Jamaica, Martinica, Puerto Rico, Santa Lucía, San Vicente, Trinidad y Tobago), Argentina, Bahamas, Belice, Bolivia, Brasil, Colombia, Costa Rica, Ecuador, El Salvador, Guayana Francesa, Guatemala, Guyana, Honduras, México (Distrito Federal, Chiapas, Colima, Guerrero, Jalisco, Oaxaca, Puebla, San Luis Potosí, Tabasco, Tamaulipas y Veracruz), Nicaragua, Panamá, Perú y Venezuela. Además, ha sido reportada en Barbados, Islas Galápagos, Montserrat e Islas Vírgenes (Santo Tomás y Santa Cruz) (Bartlett et al., 2014; Kennedy \& Bartlett, 2014). En la Argentina, sus poblaciones prevalecen en zonas tropicales, al norte de los $26^{\circ} \mathrm{S}$, particularmente en las provincias de Tucumán, Chaco y Misiones (Remes Lenicov et al., 2014a).

Recientemente, Kennedy \& Bartlett (2014) han redefinido a Caenodelphax como un género monotípico y describen e ilustran profusamente a $C$. teapae. Asimismo, estos autores citan diversos hospedantes para C. teapae: Axonopus compressus (Sw.) P. Beauv., Crotalaria sp., Cucurbita maxima Duch., Cymbopogon citratus (DC.) Stapf., Cynodon dactylon L. (Pers.), Daucus sp., Paspalum notatum Flüggé, Phaseolus vulgaris L., Saccharum sp. y Urochloa plantaginea (Link) R. Webster (Kennedy \& Bartlett, 2014). Sobre esta última se ha demostrado la actividad vectora de $C$. teapae como transmisor del tenuivirus Urochloa hoja blanca virus (UHBV) en Costa Rica (De Miranda et al., 2001).

La presencia de C. teapae en áreas afectadas con el mal de Río Cuarto, en donde la especie vectora más importante estaba ausente o en muy baja frecuencia, permite suponer que dicha especie actúa como vectora y reservorio del MRCV en la naturaleza, con su consecuente implicancia epidemiológica. El objetivo del trabajo fue determinar la capacidad de poblaciones naturales de $C$. teapae para transmitir el MRCV bajo condiciones experimentales.

\section{MATERIALES Y MÉTODOS}

\section{Muestreos de delfácidos}

Entre los años 2003 y 2006 se realizaron muestreos de delfácidos en las provincias de Tucumán y Salta. Éstos fueron efectuados sobre comunidades de poáceas naturales $(C$. dactylon y Digitaria spp. como especies predominantes) en Padilla, departamento Famaillá, provincia de Tucumán (27 $00^{\prime}$ 43,7" S; 6522' 29,8" O; 375 m s.n.m.) en 2003 (marzo y julio), 2004 (marzo, junio y septiembre), 2005 (febrero y octubre) y 2006 (julio), y en Cerrillos, departamento Cerrillos, provincia de Salta (24 53' 3,30" S; 65²8' 57,47" O; 1243 m s.n.m.) en 2003 (mayo) y 2004 (marzo).

En cada localidad y fecha se muestrearon dos lotes cercanos entre sí, recogiendo cuatro muestras por lote. Los muestreos se realizaron empleando una red entomológica de arrastre, con un esfuerzo de 50 golpes de red a pasos-hombre. En total se recogieron 64 muestras en Tucumán y 24 en Salta. Los delfácidos capturados fueron dispuestos en macetas provistas con tubos cilíndricos de acetato o en recipientes preparados a tal efecto, que contenían plantas jóvenes de trigo y/o cebada, y trasladados a laboratorio. Luego de ser tratados con gas carbónico y con ayuda de una lupa estereoscópica binocular, los insectos fueron identificados mediante clave taxonómica (Remes Lenicov \& Virla, 1999).

\section{Transmisión experimental}

Una vez identificados, 116 ejemplares adultos (machos y hembras), de C. teapae fueron sometidos a pruebas de transmisión del MRCV para la determinación de la infectividad. Cada individuo fue colocado en el interior de un tubo de vidrio de $90 \mathrm{~mm}$ de alto por $12 \mathrm{~mm}$ de diámetro y por un 
período de $24 \mathrm{~h}$, en contacto con una plántula de trigo cv. ProINTA Federal al estado de coleoptile. Este cultivar ha demostrado ser muy susceptible a la infección del MRCV y es empleado comúnmente como indicador en las pruebas de transmisión con D. kuscheli (Truol et al., 2001), especialmente por la rápida manifestación de los síntomas de la virosis. Las plántulas fueron obtenidas a partir de semillas puestas a germinar en macetas de tierra fértil, extraídas cuidadosamente a los 4-5 días y colocadas en el interior de cada tubo de vidrio, en contacto con unas gotas de agua a los fines de mantener la hidratación de las raíces. El extremo libre del tubo fue ocluido con un trozo de algodón. Los tubos así acondicionados fueron llevados a un fitotrón Conviron $₫$ con condiciones controladas de temperatura $\left(24{ }^{\circ} \mathrm{C} \pm 1\right)$, humedad $(65 \pm 5 \%)$ y fotoperíodo (16 h de luz). Una vez finalizado el período de alimentación, las plantas expuestas a los posibles insectos vectores fueron trasplantadas a macetas individuales, llevadas a invernáculo y observadas periódicamente hasta la manifestación de síntomas de la enfermedad. Todos los insectos probados fueron almacenados a $-70{ }^{\circ} \mathrm{C}$ para la posterior detección del MRCV y corroboración de identificación.

\section{Detección del MRCV en plantas inoculadas}

Las plantas inoculadas fueron sometidas a una doble prueba para confirmar la presencia del MRCV: serología y microscopía electrónica de transmisión.

Para la detección serológica del virus se empleó la técnica de DAS-ELISA (Clark \& Adams, 1977) a partir de muestras de hojas extraídas y con el empleo de reactivos específicos para el MRCV (Giménez Pecci et al., 1986; Rodríguez Pardina et al., 1998). Como controles en las pruebas serológicas se utilizaron hojas de plantas de trigo sanas e infectadas con un aislamiento del MRCV transmitido por D. kuscheli. El porcentaje de insectos transmisores del virus (infectivos) se determinó mediante el cociente entre el número de plantas seropositivas y el número total de plantas evaluadas, multiplicado por 100.

Pequeñas porciones de hoja de trigo con síntomas fueron fijadas, deshidratadas e incluidas en resina Spurr ${ }^{\circledR}$ para obtener cortes ultrafinos en ultramicrótomo. Posteriormente fueron teñidos con acetato de uranilo al $2 \%$ y citrato de plomo y observadas en un microscopio electrónico de transmisión JEOL JEM 1200 EX II en magnificaciones de 10000X. La presencia de partículas virales y posibles alteraciones fueron analizadas y comparadas con las alteraciones descriptas para diversos aislamientos del MRCV (Nome et al., 1981; Arneodo et al., 2002b).

\section{Detección del MRCV en insectos}

Un total de 64 ejemplares de C. teapae fueron analizados mediante DAS-ELISA. Para la detección serológica del MRCV en los insectos se siguió el protocolo empleado para tejidos vegetales. Los ejemplares fueron macerados individualmente en $200 \mu \mathrm{l}$ de tampón de extracción en un microtubo Eppendorf®. Como testigos se emplearon extractos de D. kuscheli provenientes de 10 ejemplares que no fueron expuestos al inóculo (avirulíferos) y extractos de 10 ejemplares previamente analizados y con altos valores de absorbancia (virulíferos). Las concentraciones de la inmuno- $\gamma$-globulina y de su conjugado enzimático fueron las utilizadas habitualmente para los tejidos vegetales (Giménez Pecci et al., 1986; Rodríguez Pardina et al., 1998). En cada celda de la inmunoplaca se sembraron $100 \mu \mathrm{l}$ de extracto. Se consideraron virulíferos aquellos insectos que registraron una lectura de absorbancia mayor o igual a la media de los testigos avirulíferos, más tres veces su desvío estándar. El porcentaje de insectos virulíferos se determinó mediante el cociente entre el número de insectos seropositivos y el número total de insectos procesados, multiplicado por 100.

\section{RESULTADOS Y DISCUSIÓN}

Mediante la captura de insectos en el campo y la posterior realización de pruebas de transmisión en laboratorio empleando hospedantes susceptibles, fue posible determinar la capacidad de $C$. teapae para transmitir el MRCV. Un total de 115 ejemplares de C. teapae se capturaron en Tucumán en los muestreos realizados en julio de 2003 $(n=69)$, junio $(n=29)$ y septiembre $(n=6)$ de 2004 , febrero $(n=3)$ y octubre $(n=7)$ de 2005 y julio de $2006(n=1)$. En Salta se capturó un ejemplar en marzo de 2004.

Del total de ejemplares de $C$. teapae recuperados de las pruebas de transmisión $(n=113)$ y de los analizados serológicamente $(n=64)$, sólo fueron detectados insectos infectivos $(10,3 \%$ ) y virulíferos $(37,5 \%)$ en aquellos procedentes de los muestreos realizados en Tucumán durante junio de 2004 (Tabla 1). El único ejemplar capturado en Salta no resultó infectivo ni virulífero. La infectividad hallada para $C$. teapae fue superior a la registrada para otros vectores naturales como D. kuscheli (entre 0,5 y 8,3\%) (Ornaghi et al., 1993b) y 
Tabla 1. Porcentaje de ejemplares de Caenodelphax teapae transmisores (infectivos) y portadores (virulíferos) del MRCV provenientes de diferentes localidades y fechas de muestreo.

\begin{tabular}{ccccc}
\hline Localidad & Año & Mes & Insectos infectivos (\%) & Insectos virulíferos (\%) \\
\hline \multirow{4}{*}{ Padilla } & 2003 & Julio & $0(0 / 69)$ & $0(0 / 44)$ \\
& 2004 & Junio & $10,3(3 / 29)$ & $37,5(3 / 8)$ \\
& 2005 & Septiembre & $0(0 / 6)$ & $0(0 / 6)$ \\
\multirow{2}{*}{ Cerrillos } & Octubre & $0(0 / 7)$ & $0(0 / 4)$ \\
& 2006 & Julio & $0(0 / 1)$ & $0(0 / 1)$ \\
\hline
\end{tabular}

Ch. haywardi (1,9\%) (Velazquez et al., 2003). Otros estudios realizados con el vector principal en la zona endémica determinaron también valores bajos de probabilidad de transmisión tanto para machos (entre 1 y $5,2 \%$ ) como para hembras (entre 1,2 y 4,6\%) (Ornaghi et al., 1999) asociados a epidemias de la virosis ocurridas con la migración de altas densidades de macrópteros hacia campos sembrados con maíz (Ornaghi et al., 1993a). La proporción de insectos virulíferos capaz de transmitir el virus $(10,3 / 37,5=0,27)$ estuvo dentro de lo hallado para las formas macrópteras del vector principal (entre 0,21 y 0,42 para machos, y entre 0,16 y 0,31 para hembras) (Ornaghi et al., 1999). Velazquez et al. (2003) hallaron para Ch. haywardi una baja proporción de virulíferos provenientes de campo que transmitieron el virus $(0,10)$, probablemente debido en parte a un incompleto período de incubación del MRCV en el insecto.

Los primeros síntomas en las plantas inoculadas se manifestaron aproximadamente a las 4 semanas luego de la exposición a los insectos, en forma de hojas de color verde más oscuro, rigidez y forma de tirabuzón. Estos síntomas, así como el tiempo transcurrido entre la inoculación y la aparición de éstos, no difirieron de los observados en plantas infectadas provenientes de campo (Rodríguez Pardina et al., 1998) y de infecciones realizadas en laboratorio por D. kuscheli (Truol et al., 2001) y por Ch. haywardi proveniente de campo (Velazquez et al., 2003). Todas las plantas inoculadas sintomáticas presentaron reacción positiva en presencia del antisuero del MRCV. Por otra parte, los valores de absorbancia hallados para las plantas sintomáticas se asemejaron a los obtenidos por Giménez Pecci et al. (1997), quienes reportaron valores leves a moderadamente altos en cereales infectados provenientes de campo.

La microscopía electrónica de transmisión reveló la presencia de viroplasmas y viriones de 60-70 $\mathrm{nm}$ de diámetro en el citoplasma de células del tejido floemático. Las características y el tamaño de las partículas observadas fueron similares a las descriptas por otros autores para el MRCV
(Nome et al., 1981; Truol et al., 2001; Arneodo et al., 2002b).

La presencia de C. teapae en el NOA donde está presente el MRCV, advierte también sobre la posibilidad que este delfácido esté involucrado en la transmisión de la enfermedad. Al igual que $D$. kuscheli, Ch. haywardi, M. propinqua, P. maidis, $P$. tigrinus y $T$. orizicolus, podría cumplir las funciones de reservorio y de agente dispersor del virus en la naturaleza.

\section{CONCLUSIONES}

La reacción del virus en las pruebas serológicas realizadas a insectos y plantas infectadas, así como la sintomatología observada en éstas y la presencia de agregados de partículas virales en células floemáticas, confirmaron que $C$. teapae tuvo la capacidad de transmitir el MRCV desde poblaciones naturales capturadas en la provincia de Tucumán a trigo. Los resultados permiten suponer el rol de C. teapae como vector y reservorio del MRCV en la naturaleza.

\section{AGRADECIMIENTOS}

Al INTA, al FONCyT, a la Agencia Córdoba Ciencia (Gobierno de la Provincia de Córdoba), al CABBIO y al CONICET. Trabajo realizado con el financiamiento de: Proyecto PICT 1998 FONCyT $N^{\circ}$ 084416, Proyecto CABBIO-FONCyT No 0006 y Proyecto Estratégico Nacional de INTA de Siembra Directa N ${ }^{\circ}$ 52-522103-14011.

\section{BIBLIOGRAFÍA}

Argüello Caro, E.B.; G.A. Maroniche, A.D. Dumón, M.B. Sagadín, M. Del Vas and G. Truol, 2013. High viral load in the planthopper vector Delphacodes kuscheli (Hemiptera: Delphacidae) is associated with successful transmission of Mal de Río Cuarto Virus. Annals of the 
Entomological Society of America 106(1):93-99.

Arneodo, J.D.; F.A. Guzmán, L.R. Conci and G.A. Truol, 2002a. Transmission features of Mal de Río Cuarto virus in wheat by its planthopper vector Delphacodes kuscheli. Annals of Applied Biology 141:195-200.

Arneodo, J.; E. Lorenzo, I. Laguna, G. Abdala and G. Truol, 2002b. Cytopathological characterization of Mal de Río Cuarto virus (MRCV, Fijivirus) in corn, wheat and barley. Fitopatologia Brasileira 27:298-302.

Bartlett, C.R.; L.B. O'Brien and S.W. Wilson, 2014. A review of the planthoppers (Hemiptera: Fulgoroidea) of the United States. Memoirs of the American Entomological Society 50:1-287.

Boito, G.T. 2005. Rol de los cereales de invierno en la epidemiología del "Mal de Río Cuarto". Tesis para optar al Grado Académico de Doctor en Ciencias Agropecuarias. Facultad de Ciencias Agropecuarias, Universidad Nacional de Córdoba, 110 pp.

Brentassi, M.E. y A.M.M. de Remes Lenicov, 1999. Oviposición de Delphacodes kuscheli (Homoptera-Delphacidae) sobre plantas de cebada en condiciones de laboratorio. Revista de la Facultad de Agronomía 104(1): 67-74.

Clark, M.F. and A.N. Adams, 1977. Characteristics of microplates methods of Enzyme Linked Immunosorbent Assay for detection of plant viruses. Journal General of Virology 34:475-483.

Conci, L.R.; M. Valdivia y S. Nome, 1992. Prospección del virus del enanismo rugoso del maíz (MRDV, "Mal de Río IV") en malezas hospedantes mediante la técnica de ELISA y de IEM. Fitopatología 27:26-32.

De Miranda, J.R.; M. Muñoz, R. Wu and A.M. Espinoza, 2001. Phylogenetic placement of a Novel Tenuivirus from the grass Urochloa plantaginea. Virus Genes 22(3):329-333.

Distéfano, A.J.; L.R. Conci, M. Muñoz Hidalgo, F.A. Guzmán, H.E. Hopp and M. Del Vas, 2002. Sequence analysis of genome segments S4 and S8 of Mal de Río Cuarto virus (MRCV): evidence that the virus should be a separate Fijivirus species. Archives of Virology 147:1699-1709.

Francki, R.I.; R.G. Milne and T. Hatta, 1985. Atlas of plant viruses. Vol. I. CRC Press, Inc. 222 pp.

Giménez Pecci, M.P.; I.G. Laguna, E. Dagoberto y P.E. Rodríguez Pardina, 1997. Detección del virus del Mal de Río Cuarto (MRCV) en triticale (Triticum x Secale) y en cebadilla criolla (Bromus unioloides H.B.K.). En: IX Congreso Latinoamericano de Fitopatología. Montevideo, Uruguay, pp. 89.

Giménez Pecci, M.P.; S.F. Nome y R.G. Milne, 1986. Purificación y obtención de antisuero de las partículas del virus causal del mal de Río IV. En: II Congreso Argentino de Virología. Córdoba, Argentina, pp. 23.
Kennedy, A.C. and C.R. Bartlett, 2014. Systematics of Caenodelphax Fennah (Hemiptera: Fulgoroidea: Delphacidae) and description of the new genus Flavoclypeus. Transactions American Entomological Society 140:17-65.

Laguna I.G. y M.P. Giménez Pecci, 2004. Enfermedades causadas por virus. Cultivo del maíz en la Argentina. IDIA XXI, Año IV, N 6, pp. 158-162.

Laguna, I.G.; M.P. Giménez Pecci, P.S. Herrera, C. Borgogno, J. Ornaghi y P. Rodríguez Pardina, 2000. Rol de los cereales de invierno y verano en la epidemiología del virus del mal de Río Cuarto (Provincia de Córdoba, Argentina). Fitopatología 35:41-49.

Laguna, I.G.; A.M. de Remes Lenicov, E. Virla, A.O. Avila, M.P. Giménez Pecci, P. Herrera, J. Garay, D. Ploper y R. Mariani, 2002. Difusión del virus del Mal de Río Cuarto (MRCV) del maíz, su vector, delfácidos asociados y huéspedes alternativos en la Argentina. Revista de la Sociedad Entomológica Argentina 61:87-97.

Lenardón, S.L.; G.J. March, S.F. Nome and J.A. Ornaghi, 1998. Recent outbreak of "Mal de Río Cuarto virus" on corn in Argentina. Plant Disease 82:448.

Lenardón, S.L.; G.J. March y J.A. Ornaghi, 1999. Virus del Mal de Río Cuarto en maíz. En: Lenardón, S.L. (Ed.). Enfermedades causadas por virus y fitoplasmas en cultivos extensivos - intensivos. Proyecto de Investigaciones en Fitovirología. IFFIVE, INTA, JICA. Hoja Informativa. Maíz № 2, 10 pp.

March, G.J.; M. Balzarini, J.A. Ornaghi, J.E. Beviacqua and A. Marinelli, 1995. Predictive model for "Mal de Río Cuarto" disease intensity. Plant Disease 79:10511053.

Mattio, M.F.; A. Cassol, A.M. de Remes Lenicov y G. Truol, 2008. Tagosodes orizicolus: nuevo vector potencial del Mal de Río Cuarto virus. Tropical Plant Pathology 33(3):237-240

Mattio, M.F.; P.D. Velazquez, A. Cassol, V. Alemandri y G. Truol, 2005. Toya propinqua Fieber como vector natural del Mal de Río Cuarto virus (MRCV). En: VI Congreso Argentino de Entomología. San Miguel de Tucumán, Argentina. Resumen N³49. p. 253.

Nome, S.F.; S.L. Lenardón, B.C. Raju, I.G. Laguna, S.K. Lowe and D. Docampo, 1981. Association of Reovirus-like particles with "Enfermedad de Río Cuarto" of maize in Argentina. Phytopathologische Zeitschrift 101:7-15

Ornaghi, J.A.; G. Boito, G. Sánchez, G. March and J.E. Beviaqcua, 1993a. Studies on the populations of Delphacodes kuscheli Fennah in different years and agricultural areas. Journal of Genetics \& Breeding 47:277-282.

Ornaghi, J.A.; G.J. March, G.T. Boito, A. Marinelli, J.E. Beviacqua, J. Giuggia and S.L. Lenardón, 1999. Infectivity in natural populations of Delphacodes kuscheli vec- 
tor of "mal de Río Cuarto" virus. Maydica 44: 219-223.

Ornaghi, J.A.; A.D. Marinelli, G.J. March, G.T. Boito y P. Rodríguez Pardina, 1993b. Transmisión del virus causal del mal de Río Cuarto por Delphacodes kuscheli Fennah a cultivos y malezas. En: Workshop "Mal de Río Cuarto del Maíz". INTA-IFFIVE. Córdoba, Argentina, 23-25 de junio. pp. 41-42.

Remes Lenicov, A.M. Marino de; A. Maciá, R. Mariani, A. Foieri, M.E. Brentassi, A. Toledo, M.F. Rossi Batiz y S. Paradell, 2014a. Diversidad de Fulgoromorpha (Insecta-Hemiptera) y fluctuaciones poblacionales de especies vectoras asociadas con el agrosistema maíz en tres áreas agrológicas del NOA. En: X Congreso Nacional de Maíz. Rosario, Santa Fe, Argentina, 5-8 de septiembre. 4 pp.

Remes Lenicov, A.M. Marino de and G. Varela, 2014b. A new genus and species of Delphacini (Hemiptera: Fulgoroidea: Delphacidae) from Argentina. Zootaxa 3861(2)177-184.

Remes Lenicov, A.M.M. de; A. Tesón, E. Dagoberto y N. Huguet, 1985. Hallazgo de uno de los vectores del Mal de Río Cuarto en maíz. Gaceta Agronómica 5(25):251-258.

Remes Lenicov, A.M.M. de y E. Virla, 1999. Delfácidos asociados al cultivo de maíz en la República Argentina (Insecta-Homoptera-Delphacidae). Revista de la Facultad de Agronomía (La Plata) 104(1):1-15.

Remes Lenicov, A.M.M. de; E. Virla y E. Dagoberto, 1991. Cambios estacionales en la población del vector del "Mal de Río Cuarto" del maíz (Delphacodes kuscheli Fennah, 1955) en cultivos de avena y sus malezas circundantes en Sampacho, Córdoba (Insecta-Homoptera-Fulgoroidea). En: Taller de Actualización sobre Mal de Río Cuarto. INTA, CIMMYT. pp. 116-129.

Rodríguez Pardina, P.E., M.P. Giménez Pecci, I.G. Laguna, E. Dagoberto and G. Truol, 1998. Wheat: a new natural host for the Mal de Río Cuarto Virus in the endemic disease area, Río Cuarto, Córdoba province,
Argentina. Plant Disease 82:149-152.

Truol, G.A.; T. Usugi, J. Hirao, J.D. Arneodo, M.P. Giménez Pecci y I.G. Laguna, 2001. Transmisión experimental del virus del mal de Río Cuarto por Delphacodes kuscheli. Fitopatologia Brasileira 26:39-44.

Velazquez, P.D., 2010. Transmisión experimental y detección del virus del Mal de Río Cuarto (MRCV) en diferentes especies de delfácidos (Insecta: Hemiptera). Tesis de Doctorado, Facultad de Ciencias Exactas, Físicas y Naturales, Universidad Nacional de Córdoba, Argentina. $162 \mathrm{pp}$.

Velazquez, P.D.; J.D. Arneodo, F.A. Guzmán, L.R. Conci and G.A. Truol, 2003. Delphacodes haywardi Muir, a new natural vector of Mal de Río Cuarto virus in Argentina. Journal of Phytopathology 151:669-672.

Velazquez, P.D.; L.R. Conci y G. Truol, 2001. Toya propinqua (Hemiptera: Delphacidae): especie vectora del virus del mal de Río Cuarto (MRCV) en transmisiones experimentales. Fitopatologia Brasileira 26(S):542.

Velazquez, P.D.; F.A. Guzmán, L.R. Conci, A.M. de Remes Lenicov y G.A. Truol, 2006. Pyrophagus tigrinus Remes Lenicov \& Varela (Hemiptera: Delphacidae), nuevo vector del Mal de Río Cuarto virus (MRCV, Fijivirus) en condiciones experimentales. Agriscientia 23(1):9-14.

Virla, E.G.; I. Miotti, M.P. Giménez Pecci, P. Carpane y G. Laguna, 2003. Peregrinus maidis (Hemiptera: Delphacidae), nuevo vector experimental del "Mal de Río Cuarto" del maíz. En: XX Jornadas Científicas de la Asociación de Biología de Tucumán. Tafí del Valle, Tucumán, Argentina, pp. 54-55.

Virla, E. y A.M.M. de Remes Lenicov, 1991. Ciclo de vida de Delphacodes kucheli criado sobre diferentes hospedantes en condiciones de laboratorio (InsectaHomoptera-Delphacidae). En: Taller de Actualización sobre Mal de Río Cuarto. INTA, CIMMYT. pp. 104-115. 